\title{
A Review on Various IOT Analytics Techniques for Bridge Failure Detection in Fog Computing
}

\author{
Suneha Amar \\ M.Tech Scholar \\ Department of Computer Science \& Engineering \\ GNDU Regional Campus, Jalandhar, \\ Punjab, India
}

\author{
Pankaj Deep Kaur, PhD \\ Assistant Professor \\ Department of Computer Science \& Engineering \\ GNDU Regional Campus, Jalandhar, \\ Punjab, India
}

\begin{abstract}
IoT analytics is the alteration of enormous quantities of information in significant styles and rules. To expect describing the previous in addition to calculating the long term via data analysis. IoT analytics is a multi-disciplinary area which mixes, machine learning, research, data source technologies and artificial intelligence. IOT analytics is usually achieved in several stages of development: Business enterprise knowing, Information knowing, Information preparing, Acting, Review, and Deployment. There are various IOT analytics methods when Affiliation, Distinction, Clustering, Sensation problems System and Regression. This research work presents different IOT analytics algorithms intended for effectively mining the particular health-related facts set. IOT analytics algorithms have grown to be favorite every day live apps including breach prognosis procedure, diabetes mellitus exploration, e-mail spam distinction etc. In this paper we discuss about the various techniques of IOT and also IOT analytics techniques for bridge failure detection IOTs. The overall objective of this paper is to bridge failure detection in IOT
\end{abstract}

\section{Keywords}

Fog computing; bridge failure detection;Internet Of Things (IOT).

\section{INTRODUCTION}

The particular fog Computing paradigm, delineate the item has the properties, plus that surrounding method in which allows for Fog services. This areas necessitates an in depth take a look at a handful of necessary uses plus companies appealing in which substantiate some of our talk throughout inclination with the unique Fog for the reason that real section of method necessary for your guide for the Internet of Things. Fog computing will be an extremely virtualized podium that delivers estimate, storage area, as well as networking companies amid conclude tools plus traditional Damage Computing Information Stores, usually, yet not simply placed close to network.

\subsection{Need of Fog}

The actual errors Computing paradigm, delineate that contains the homes, and also that surrounding program by which encourages Errors services. That segments necessitates a close consider a few vital works by using and also suppliers appealing by which substantiate your discussion with preference on the particular Errors ever since the genuine bit of program required for the exact guide for the Internet associated with Things. Errors computing will likely be an extremely virtualized podium giving you determine, safekeeping, as well as networking suppliers among finish gear and also regular Foreign Computing Data files Centres, commonly, yet not simply situated all around network. As soon as strategies and also devices associated with IOT are usually reading good linked to lenders existence, latest Cloud computing paradigm might barely fulfill its needs associated with range of motion service, area awareness and also very low latency. Fog computing is usually recommended to address the above problem. When Fog computing is usually put in place at the edge of a circle, it offers a superior very low latency, area awareness, and also helps quality-ofservices (QoS) for internet streaming and also real-time software [2]. Common these include industrial automation, transfer, and also systems associated with receptors and also actuators. In addition, this specific fresh national infrastructure can handle heterogeneity while Fog gadgets involve end-user gadgets, gain access to issues, border routers in addition to switches. The Fog paradigm is effectively situated with regard to real time massive information analytics, can handle densely spread information series issues, and supplies strengths throughout entertainment, advertising, personal computing and various applications. Fog computing runs the particular paradigm on the border of your network. although fog in addition to fog up making use of similar resources(networking, computing, storage) in addition to write about many of the similar system in addition to attribute(virtualization, multi-tenancy) the particular file format is a low trivial one in this there are many fundamental disparities coming with the explanation fog computing created: to address in addition to providers that won't accommodate the particular paradigm with fog up.

\subsection{Application of Fog Computing}

- Connected Cars: Fog computing is the best for the attached vehicles(CV) simply because live friendships can certainly make connection involving cars, accessibility factors plus site visitors lighting fixtures because protected plus useful because possible. Training video dslr camera in which senses a good emergency flashing lighting fixtures might routinely adjust neighborhood lighting fixtures to spread out lanes regarding the passenger truck prush out a traffic. Clever neighborhood lighting fixtures interact in your community having receptors plus diagnose existence with men and women plus motorcyclist, plus measure the gap plus swiftness with drawing near vehicles. Seeing that demonstrated within Number, intelligent lighting effects activates each indicator determines motion plus buttons away because site visitors passes. Bordering smart lighting fixtures serving as Fog systems put together to make natural site visitors trend plus send out warning signals in order to drawing near vehicles. Wifi accessibility factors for instance Wi-Fi, 3G, road-side models plus smart site visitors lights are used along the 
roads. Vehicles-to Motor vehicle, auto to gain access to factors, plus accessibility factors to gain access to factors friendships enhance the employment of that circumstances [5][4].

- Smart Grids: Fog computing let speedy, machine in order to machine (M2M) handshakes plus human in order to machine friendships (HMI) which will work with synergy while using cloud. Electrical power weight controlling programs may run in network edge units, just like wise feets plus micro-grids Determined by vitality demand, accessibility and the smallest cost, these units instantly switch to option powers similar to sun plus wind. Since revealed inside Determine, Fog debt collectors on the edge method the info earned by way of power company devices plus units, plus situation handle commands towards actuators. In addition they filtration system the info for being ingested in the area, plus send the remainder towards larger layers with regard to visualization, real-time stories plus transactional analytics. Fog facilitates ephemeral safe-keeping at the smallest rate in order to semi-permanent safe-keeping at the very best tier. World wide insurance coverage will be furnished by a Fog up using company intellect analytics [1] [7].

- Health care: The particular cloud computing sell for health care is expected to succeed in $\$ 5.4$ billion by simply 2017 along with fog computing will permit this with a more local level.

- Wireless Sensor and Actuator Networks: Regular instant indicator cpa networks don't succeed with purposes that will exceed detecting along with tracking, however call for actuators in order to have to put out physical measures just like opening, final or perhaps carrying sensors. Within this circumstances, actuators becoming Errors equipment may command a statistic method by itself, the soundness as well as oscillatory behaviors simply by making a closed-loop system. As an example, while in the circumstances regarding self-maintaining locomotives, indicator tracking with locomotives ballbearing may identify high temperature ranges, letting purposes to send out an automated responsive to a educate operator to halt a educate at up coming place to get urgent situation repair and steer clear of possibilities derailment.

Smart Cities: Fog computing could obtain indicator details about many amounts of pursuits regarding places as well as combine every one of the mutually unbiased community organisations within. The actual uses of this kind of circumstance are triggered by simply wireless alerts used in order to gauge heat range, moisture content, or perhaps amounts of many un wanted gas within your building atmosphere. In cases like this, data is usually swapped among all alerts within the ground, and numbers is usually bundled to make reliable measurements. Receptors use distributed selection as well as triggering at Fog equipment in order to interact with data. The system elements might band together in order to reduce a heat range inject outside or perhaps wide open windows. Air conditioning equipment can can get rid of humidity with the atmosphere or perhaps boost the humidity. Receptors might also locate as well as interact with actions (e.g., by simply rotating light about or perhaps off). Fog equipment can be given at every flooring and may work with others about higher level regarding actuation. With Fog computing applied in this kind of circumstance, good houses can preserve their materials, internal and external surroundings to save power, mineral water as well as other assets [2]

\subsection{Internet of Things (IOT)}

The Internet of Things (IOT) paradigm is dependent on sensible along with self applied establishing nodes(things) interconnected inside of a powerful along with world wide community infrastructure. That shows the single most troublesome technological innovation, empowering allpervasive along with persistent computing scenarios. IOT is often seen real world modest factors, extensively allocated, together with restricted hard drive along with control potential, which will entail concerns regarding dependability, overall performance, safety, along with privacy. The Worldwide-web of Elements (IOT) is actually circumstances where physical objects, family pets and also individuals are provided by unique identifiers along with the opportunity to shift files spanning a community with out requiring human-to-human and also human-to-computer interaction. IOT has changed from the unity of cellular technological innovation, microelectromechanical systems (MEMS) along with the Internet. Your World-wide-web of Elements, at times World-wide-web of Everything) is definitely the community of real physical objects and also "factors" inlayed together with consumer electronics, software package, receptors, along with connectivity allow physical objects to files together with the company, driver and/or some other associated products in accordance with the structure of Overseas Telecommunication Union's Worldwide Specifications Initiative. Your Worldwide-web of Elements will allow physical objects being sensed along with governed a little bit all around established community structure, producing opportunities to get more detailed lead integrated, relating to the real community along with computer-based systems, along with resulting in increased performance, precision along with global financial benefit.

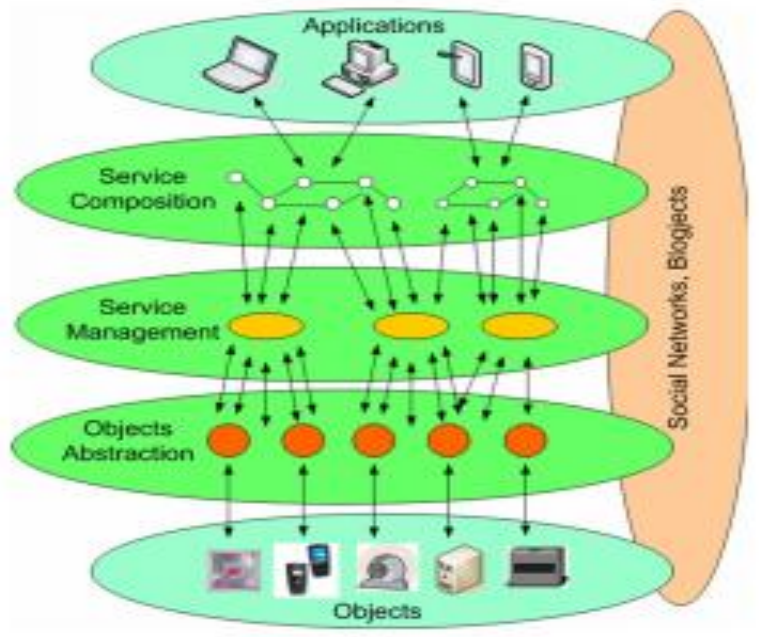

Fig 1: IOT Paradigm

\subsection{Fog Computing - A Natural Bridge between Cloud and IOT}

A new craze may possibly be observed with joint relative by using fog up - it absolutely was a sluggish start December 2013, talking over about a new concept which driving unique brand established the actual office space intended for sent out fog up models. Around February 2015 we might describe Errors Computing while important bridge involving the 
limitless electric power in the Impair in addition to a limitless amount of intelligent edge details, conventionally conglomerated with IOT concept. What exactly is transpired by using Errors Computing in this particular really short period? Impair computing affirmed a lot of technology in addition to market institutions, remaining involved with all key technological innovation trends, out of Major Details in addition to Analytics, to be able to Flexibility, M2M, in addition to Net with Everything. Around concurrent using this, IOT principle gets the intense development, obtaining while main power plant huge raising of knowledge lists supplied by an increasing number of people and big number of intelligent devices. Errors computing vision, made upon thinking about having fog up computing capabilities to your edge in the network, very much deeper to your growing amount of person units which have been having fog up providers in addition to generating the actual progressively more significant quantity of data. According 'cisco', Errors Computing stretches the actual fog up computing paradigm to your edge in the network. When errors in addition to fog up use the same computing, storage area or maybe network assets, in addition to promote most of the same systems in addition to properties, such as multi-tenancy in addition to virtualization. At this point we might talk about your Errors Computing ecosystem based on Errors conceptually extension with Impair computing - addressing within a more dense means bigger topographical spots, in addition to levels with Errors units - considerably more heterogeneous by nature, which range from end-user final, gain access to details, to be able to cpa networks edge routers in addition to switches. Given details need to be packaged regionally with smart units as an alternative to remaining directed intended for handling in the cloud. Errors computing is but one way of dealing with the demands in the ever-increasing range smaller associated units, often termed as the actual Net with Elements [1]

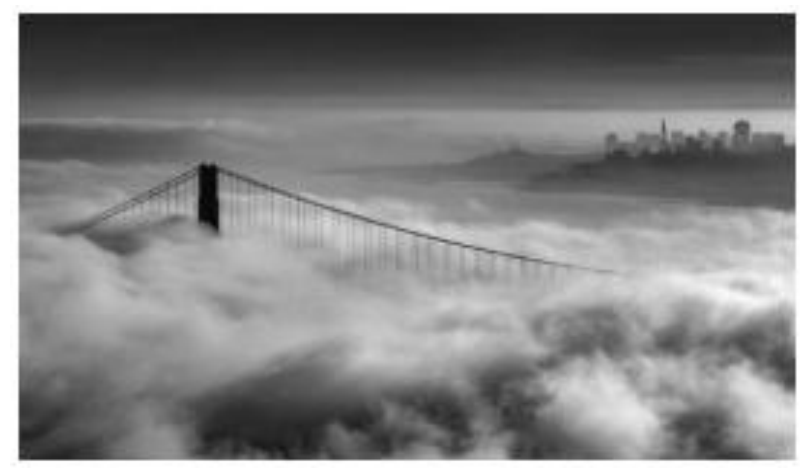

Fig 2. Bridge between fog and cloud

\section{LITERATURE REVIEW}

Manuel Diaz et al. [1] that World wide web involving Issues (IOT) is really a paradigm based on the World wide web that contains lots of interconnected systems like RFID (Radio Rate of recurrence Identification) plus WSAN (Wireless Sensor / probe plus Actor or actress Networks) so as to return information. Today's desires pertaining to greater control, supervising plus operations in several places, and also the continuous homework during this subject, possess originated the design plus introduction of a number of solutions like smart-home, smart-city plus smart-grid. Nevertheless, the restrictions involving connected units inside the IOT in terms of safe-keeping, system plus computing, plus the requirements of difficult examination, scalability, plus data admittance, require a engineering like Reasoning Computing in order to nutritional supplement this field. What's more, this IOT can certainly create copious amounts involving diverse data plus easily when there are millions of issues giving data in order to Reasoning Computing. These is definitely a transparent example of Massive Details, that Reasoning Computing desires to adopt straight into account. This kind of papers shown a survey involving intergrated, pieces: Reasoning platforms, Reasoning infrastructures plus IOT Middleware. Also, many intergrated, suggestions plus data analytics strategies are usually surveyed as well as different challenges plus start homework challenges are usually pointed out. Tuan Nguyen Gia et al. [2] enhanced World-wide-web regarding Issues (IOT) is often a paradigm in line with the World-wideweb that will contains quite a few interlocked systems for instance RFID (Radio Regularity Identification) in addition to WSAN (Wireless Warning in addition to Actor or actress Networks) so that you can exchange information. Today's wants pertaining to much better command, supervising in addition to managing in several spots, and also the ongoing exploration with this area, possess all began the appearance in addition to introduction of various methods for instance smart-home, smart-city in addition to smart-grid. Even so, the restrictions regarding related gadgets from the IOT regarding storage space, system in addition to computing, in addition to the requirements complex evaluation, scalability, in addition to files entry, degree of technological innovation for instance Fog up Computing so that you can health supplement this kind of field. What's more, your IOT can bring in massive amounts regarding assorted files in addition to quickly when there are millions of elements eating files so that you can Fog up Computing. Ppos is usually a particular example of Massive Files, that will Fog up Computing wants to consider within account. The following papers introduced market research regarding intergrated, pieces: Fog up websites, Fog up infrastructures in addition to IOT Middleware. Additionally, a number of intergrated, plans in addition to files analytics approaches are generally surveyed and also distinct challenges in addition to open up exploration challenges are generally sharpened out..Alessio Botta et al. [3] introduced Reasoning computing plus Web regarding Items (IOT) will be a couple of different technologies which tend to be previously component of the life. The usage plus use is predicted to be increasingly more persistent, driving them to important components from the Potential Internet. Some sort of new paradigm exactly where Reasoning plus IOT will be merged jointly is definitely predicted while disruptive and also as a enabler regarding numerous software scenarios. On this document, most people concentration the interest around the plug-in regarding Reasoning plus IOT, which happens to be that which we phone your Reasoning IOT paradigm. Flavio Bonomi et al. [4] focused onNet regarding Things (IOT) gives above a great mind-blowing proliferation regarding endpoints. It really is troublesome in numerous ways. During this chapter most of us study those interferences, and also propose to your girlfriend the hierarchical allocated buildings which offers with the side on the multilevel for the primary nicknamed Fog Computing. Especially, most of us pay attention to the latest sizing which IOT improves Big Facts and also Analytics: the extremely allocated amount of sources with the edge. Luis M Vaquero et al. [5] represents thatimpair is actually migrating to the borders of your community, the place routers on their own could end up being the virtualization structure, in a development labelled seeing that "a haze ".Having said that, a number of other contributory technology is reaching a high level associated with maturity. Their particular interplay could significantly transfer the content and communication technology scenery in the following years, having distinct 
technologies in to a typical ground. That newspaper presents a wide meaning "a haze", knowledge technologies seeing that various seeing that impair, alarm sites, peer-to-peer sites, community virtualization performs or configuration control techniques. All of us spotlight the main problems experienced by this perhaps break-through technology amalgamation. Ivan Stojmenovic et al. [6] shows network. Just like Impair, Haze offers files, calculate, storage area, and software expert services to be able to end-users. In this post, we all complex the particular commitment and attributes of Haze computing, and evaluate it has the applications with a number of genuine conditions, such as Wise Metered, smart targeted visitors equipment and lighting with road networks and application explained networks. Most of us talk about the particular stateof-the-art associated with Haze computing and other do the job underneath a similar umbrella. Protection and privacy concerns usually are additional revealed reported by latest Haze computing paradigm. As an example, we all review an average invasion, man-in-the-middle invasion, to the debate associated with security in Haze computing. Most of us investigate stealthy top features of this invasion by way of reviewing it has the CPU and memory space usage about Haze device. Madsen, Henrik, et al. [7] considers existing paradigms throughout computing and also sets out the most crucial aspects with regards to their particular reliability. The Haze computing paradigm being a non-trivial file format of your Foreign is known as and the reliability of a sites involving clever items are discussed. Combining a reliability needs involving metered and also fog up paradigms with all the reliability needs involving sites involving sensor and also actuators it makes sense which designing a reliable Haze computing platform can be feasible. Hong, Kirak, et al. [8] that deployment associated with mobile along with indicator units is definitely creating a brand new natural environment, that is the particular Web associated with Elements (IOT) that enables a variety of future Web applications. Around this work, we all provide Cellular Errors, an advanced level selection product for future Web apps that are geospatially distributed, large-scale, along with latency-sensitive. Most of us investigate work with conditions to the selection product by using digital camera system along with associated automobile apps to point out the particular efficaciousness associated with Cellular Fog. We examine use operation by way of simulation. Zhu, Jiang, et al. [9] considered net search engine optimization within just Errors Computing context. We all apply existing options for net search engine optimization in a very work of fiction method, such that they is usually joined with exceptional awareness that is only offered in the advantage (Fog) nodes. A lot more vibrant variation towards the client's ailments (eg. multi-level position and device's computing load) will also be accomplished by using multi-level advantage specific knowledge. Therefore, any client's web page making operation is improved above that will realized just by making use of all those strategies in the net web server or even CDNs .Wang, Wenye et al. [10] smart Grid, typically called this next-generation energy procedure, is regarded as a progressive as well as evolutionary regime regarding current energy grids. More importantly, together with the incorporation regarding sophisticated computing as well as interaction technology, this Sensible Power grid is predicted to significantly enrich overall performance as well as robustness of future energy techniques by using alternative energy sources, together with distributed thinking ability as well as requirement response. Together with the quiet popular features of this Sensible Power grid, online safety emerges becoming a vital matter mainly because an incredible number of electric devices are inter-connected via interaction sites throughout vital energy services, which includes an instant affect on robustness of this sort of a widespread infrastructure. In this document, most people existing an intensive review regarding online safety troubles for that Sensible Grid. Specifically, most people center on critiquing as well as going over safety requirements, community weaknesses, invasion countermeasures, safe and sound interaction protocols as well as architectures from the Sensible Grid. Most people target use a deep familiarity with safety weaknesses as well as methods from the Sensible Power grid as well as shed light on future study directions for Sensible Power grid security.

\section{COMPARISON TABLE}

Table 1. Comparison of various IOT analytic techniques

\begin{tabular}{|l|l|l|l|l|}
\hline Ref no & \multicolumn{1}{|c|}{ Authors } & \multicolumn{1}{|c|}{ Technique } & \multicolumn{1}{|c|}{ Feature } & \multicolumn{1}{c|}{ Limitation } \\
\hline 1. & Atzori, Luigi & Internet of Things & $\begin{array}{l}\text { Enhanced communication protocols, } \\
\text { and distributed intelligence for smart } \\
\text { objects are just the most relevant. }\end{array}$ & $\begin{array}{l}\text { The current Internet paradigm, which } \\
\text { supports and has been built around } \\
\text { host-to-host communications, is now a } \\
\text { limiting factor for the current use of the } \\
\text { Internet. }\end{array}$ \\
\hline 2. & Bostock & $D^{3}$ Data-Driven & $\begin{array}{l}\text { Representational transparency improves } \\
\text { expressiveness and better integrates } \\
\text { with developer tools than prior } \\
\text { approaches }\end{array}$ & $\begin{array}{l}\text { Evolutionary optimization techniques } \\
\text { have not considered }\end{array}$ \\
\hline 3. & Buyya & Cloud computing & $\begin{array}{l}\text { Encompass both customer-driven } \\
\text { service management and computational } \\
\text { risk management to sustain Service }\end{array}$ & $\begin{array}{l}\text { Finally, we need to address regulatory } \\
\text { and legal issues, which go beyond } \\
\text { technical issues. }\end{array}$ \\
\hline
\end{tabular}




\begin{tabular}{|c|c|c|c|c|}
\hline & & & $\begin{array}{lll}\text { Level Agreement } & \text { (SLA)-oriented } \\
\text { resource allocation. } & \end{array}$ & \\
\hline 4. & Castronova & $\begin{array}{l}\text { Open Geospatial } \\
\text { Consortium (OGC) }\end{array}$ & $\begin{array}{l}\text { This approach is that the server-side } \\
\text { software follows an established standard } \\
\text { that can be leveraged and reused within } \\
\text { multiple workflow environments and } \\
\text { decision support systems }\end{array}$ & $\begin{array}{l}\text { Meta-heuristic techniques have not } \\
\text { been considered }\end{array}$ \\
\hline 5. & Christin & $\begin{array}{l}\text { Wireless Sensor } \\
\text { Networks and the } \\
\text { Internet of things }\end{array}$ & $\begin{array}{l}\text { We have selected three important task } \\
\text { assignments in order to highlight the } \\
\text { challenges emerging from the paradigm } \\
\text { adoption: Security, QOS, and } \\
\text { configuration management. }\end{array}$ & $\begin{array}{l}\text { Their analysis revealed that the } \\
\text { solutions currently deployed in the } \\
\text { Internet are not suitable for the limited } \\
\text { sensor node resources } \\
\text { and consequently. }\end{array}$ \\
\hline 6. & Christodoulou & $\begin{array}{l}\text { Water Loss } \\
\text { Detection }\end{array}$ & $\begin{array}{l}\text { The improvement of the performance } \\
\text { and the reduction of the power } \\
\text { consumption of the network. }\end{array}$ & $\begin{array}{l}\text { The risk of failure for } \\
\text { each segment of the pipe network based } \\
\text { on rolling historical data. }\end{array}$ \\
\hline 7. & Brandon P & $\begin{array}{l}\text { Real-time } \\
\text { environmental } \\
\text { sensor data }\end{array}$ & $\begin{array}{l}\text { To make significant gains in the use of } \\
\text { real-time data. }\end{array}$ & $\begin{array}{l}\text { The results are not scalable and } \\
\text { efficient }\end{array}$ \\
\hline 8. & Dermot & $\begin{array}{l}\text { Chemo-/ Bio } \\
\text { sensing }\end{array}$ & $\begin{array}{l}\text { Enhanced communication protocols, } \\
\text { and distributed intelligence for smart } \\
\text { objects are just the most relevant. }\end{array}$ & $\begin{array}{l}\text { Finally, we need to address regulatory } \\
\text { and legal issues, which go beyond } \\
\text { technical issues. }\end{array}$ \\
\hline 9. & Díaz & $\begin{array}{l}\text { Geospatial } \\
\text { Information } \\
\text { Infrastructures }\end{array}$ & $\begin{array}{l}\text { To improve the registration of sensors } \\
\text { and the publication of their observations } \\
\text { via standards-based Web service } \\
\text { interfaces. }\end{array}$ & $\begin{array}{l}\text { Evolutionary optimization techniques } \\
\text { have not considered }\end{array}$ \\
\hline 10. & Heather E & $\begin{array}{l}\text { Hydrograph } \\
\text { modelling with real- } \\
\text { time }\end{array}$ & $\begin{array}{l}\text { The utility of the algorithm was } \\
\text { successfully tested with hydrograph data } \\
\text { collected at both a tile drain and } \\
\text { agricultural ditch, suggesting the } \\
\text { potential for general applicability of the } \\
\text { method }\end{array}$ & $\begin{array}{l}\text { The current Internet paradigm, which } \\
\text { supports and has been built around } \\
\text { host-to-host communications, is now a } \\
\text { limiting factor for the current use of the } \\
\text { Internet. }\end{array}$ \\
\hline
\end{tabular}

\section{CONCLUSION}

IOT integrates numerous units designed with detecting, recognition, control, conversation, and networking capabilities. Especially, sensors and actuators are receiving progressively more potent, a smaller amount expensive and smaller sized, helping to make their particular use everywhere.This paper represents the comparison between various IOT techniques by which we detect the air pollution in fog computing. The main Objective of this paper is to The overall objective of this paper is to bridge failure detection in IOT. Their improvement is done by use of unsupervised filtering which will be followed by IOT analytics techniques for bridge failure detection in Fog computing.

\section{REFERENCES}

[1] Díaz, Manuel, Cristian Martín, and Bartolomé Rubio. "State-of-the-art, challenges, and open issues in the integration of Internet of things and cloud computing." Journal of Network and Computer Applications 67 (2016): 99-117.

[2] Gia, Tuan Nguyen, et al. "Fog computing in healthcare internet of things: A case study on ecg feature extraction." Computer and Information Technology; Ubiquitous Computing and Communications; Dependable, Autonomic and Secure Computing; Pervasive Intelligence and Computing (CIT/IUCC/DASC/PICOM), 2015 IEEE International Conference on. IEEE, 2015.

[3] Abdelwahab, Sherif, et al. "Enabling smart cloud services through remote sensing: An internet of everything enabler." IEEE Internet of Things Journal 1.3 (2014): 276-288.

[4] Bonomi, Flavio, et al. "Fog computing: A platform for internet of things and analytics." Big Data and Internet of Things: A Roadmap for Smart Environments. Springer International Publishing, 2014. 169-186. 
[5] Vaquero, Luis M., and Luis Rodero-Merino. "Finding your way in the fog: Towards a comprehensive definition of fog computing." ACM SIGCOMM Computer Communication Review 44.5 (2014): 27-32.

[6] Stojmenovic, Ivan, and Sheng Wen. "The fog computing paradigm: Scenarios and security issues." Computer Science and Information Systems (FedCSIS), 2014 Federated Conference on. IEEE, 2014.

[7] Madsen, Henrik, et al. "Reliability in the utility computing era: Towards reliable fog computing." Systems, Signals and Image Processing (IWSSIP), 2013 20th International Conference on. IEEE, 2013.

[8] Hong, Kirak, et al. "Mobile fog: A programming model for large-scale applications on the internet of things." Proceedings of the second ACM SIGCOMM workshop on Mobile cloud computing. ACM, 2013.

[9] Zhu, Jiang, et al. "Improving web sites performance using edge servers in fog computing architecture." Service
Oriented System Engineering (SOSE), 2013 IEEE 7th International Symposium on. IEEE, 2013.

[10] Wang, Wenye, and Zhuo Lu. "Cyber security in the Smart Grid: Survey and challenges." Computer Networks 57.5 (2013): 1344-1371.

[11] Luan, Tom H., et al. "Fog computing: Focusing on mobile users at the edge." arXiv preprint arXiv:1502.01815 (2015).

[12] Stolfo, Salvatore J., Malek Ben Salem, and Angelos D. Keromytis. "Fog computing: Mitigating insider data theft attacks in the cloud." Security and Privacy Workshops (SPW), 2012 IEEE Symposium on. IEEE, 2012.

[13] F. Bonomi, "Connected vehicles, the internet of things, and fog computing," in The Eighth ACM International Workshop on Vehicular Inter- Networking (VANET), Las Vegas, USA, 2011. 